\title{
The role of mean platelet volume in patients with non-specific abdominal pain in an emergency department
}

\author{
Adil Coskun ${ }^{1}$, Irfan Yavasoglu², Gokhan Sargin ${ }^{3}$, Ismail Murat Ok ${ }^{4}$, Metin Bircan ${ }^{4}$, Mucahit Avcil $^{4}$, \\ Gurhan Kadikoylu², Zahit Bolaman² \\ ${ }^{1}$ Division of Gastroenterology, Medical Faculty, Adnan Menderes University, Aydin, Turkey \\ ${ }^{2}$ Division of Haematology, Medical Faculty, Adnan Menderes University, Aydin, Turkey \\ ${ }^{3}$ Department of Internal Medicine, Medical Faculty, Adnan Menderes University, Aydin, Turkey \\ ${ }^{4}$ Department of Emergency Medicine, Medical Faculty, Adnan Menderes University, Aydin, Turkey
}

Prz Gastroenterol 2015; 10 (3): 156-159

DOI: $10.5114 /$ pg.2015.49042

Key words: emergency department, non-specific abdominal pain, mean platelet volume.

Address for correspondence: Gokhan Sargin MD, Department of Internal Medicine, Medical Faculty, Adnan Menderes University, 09000 Aydin, Turkey, phone: 5068655990, e-mail: gokhan_sargin@hotmail.com

\begin{abstract}
Introduction: Platelets play an important role in inflammation. Mean platelet volume (MPV) may be a useful parameter for inflammatory conditions, in differentiating between non-specific abdominal pain (NSAP) and conditions requiring surgery, or early diagnosis of abdominal pain as a serious problem for emergency services.

Aim: To investigate the role of MPV on NSAP admittance to the emergency department.

Material and methods: The study consisted of 330 patients (186 female and 144 male, with mean age $49 \pm 20$ years) with NSAP and 249 patients with abdominal pain (144 female and 105 male, with mean age $49 \pm 19$ years) applied to our emergency service and enrolled to our study. Medical history, physical examination, complete blood count (CBC), and biochemical parameters were evaluated. Haematological parameters were measured by Beckman Coulter LH 780 instrument. Data were compared using Student's $t$ test, and $p<0.05$ was accepted as statistically significant.

Results: The MPV were significantly lower in patients with NSAP than in others $(p=0.025)$. There was no significant difference between both sexes with regard to MPV $(p>0.05)$. Moreover, the other haematological parameters (haemoglobin, platelet, or WBC counts) were not statistically different between both groups ( $p>0.05)$.

Conclusions: The MPV may be a useful, simple, and inexpensive parameter to diagnose or differentiate between NSAP and other conditions.
\end{abstract}

\section{Introduction}

Abdominal pain is a common presenting complaint. It accounts for up to $10 \%$ of all patients applying to the emergency department. Many different conditions may cause abdominal pain (endocrinological, haematological, neurological, inflammatory, infectious, non-abdominal, etc.). Nonspecific abdominal pain (NSAP) accounts for $50 \%$ of abdominal pain, and different surgical procedures are performed in $13-40 \%$ of patients with NSAP [1-3]. However, these methods are not easy and may cause complications.

Platelets play an important role in inflammation [4]. There are complex interactions (including proinflammatory cytokines, chemokines, platelet and endothelial activation) between inflammation and haemostasis. Inflammation increases procoagulant factors, and coagulation also augments inflammation. Platelets may also trigger inflammation through dendritic cells [5]. Increased mean platelet volume (MPV) was reported in patients with acute myocardial infarction, acute cerebral ischaemia, and transient ischaemic attack [6-8]. The MPV may be a useful parameter for inflammatory conditions [9-11].

\section{Aim}

The aim of this study is to investigate the role of MPV on NSAP admittance to the emergency department. 


\section{Material and methods}

The study enrolled 330 patients (186 female and 144 male, with mean age $49 \pm 20$ years) with NSAP and 249 patients (144 female and 105 male, with mean age $49 \pm 19$ years), who applied to our emergency service with different aetiology of abdominal pain and enrolled in this retrospective study. Thrombocytopaenia, congenital or acquired platelet dysfunctions, smoking, hypertension, hyperlipidaemia, diabetes mellitus, haematological disease, history of cerebrovascular accident, coronary heart disease, and the use of anti-coagulants and/or anti-platelet drugs were exclusion criteria for this study.

We evaluated patients via medical history, physical examination, complete blood count, biochemistry, direct $X$-ray, ultrasonography, and computed tomography. Abdominal pain was accepted as NSAP if there was no sign for other aetiology of abdominal pain. All patients gave written, informed consent for the study.

Complete blood count was performed on a Beckman Coulter LH 780 instrument. White blood cell (WBC) and platelet counts, haemoglobin, and MPV levels were evaluated. Quality controls in our laboratory documented good reproducibility of MPV measurements, with intra-assay and inter-assay coefficients of variation $\leq 2.2 \%$ on commercial controls. The reference range of this device for MPV was 6.5-11.6 fl.

\section{Statistical analysis}

The data were evaluated using SPSS (Statistical Package for Social Sciences) 13.0 statistical software. Parameters were compared using Student's $t$ test, and $p<0.05$ was accepted as statistically significant.

\section{Results}

The patients with abdominal pain were divided into two groups: NSAP and others. The causes of abdominal pain are summarised in Table I. In both groups, platelet counts were more than $100,000 / \mathrm{mm}^{3}$. The NSAP accounts for $56 \%$ of complaints of abdominal pain. Demographic and haematological parameters are shown in Table II.

The MPV values were significant lower in patients with NSAP than in the others $(p=0.025)$. There was no significant difference between both sexes for MPV $(p>0.05)$. Moreover, the other haematological parameters (haemoglobin, platelet, or WBC counts) were not statistically different between both groups $(p>0.05)$.

\section{Discussion}

In this study, MPV was significantly lower in patients with abdominal pain than in the others. But there was no significant difference for platelet counts between both groups. Increased MPV is associated with increased platelet production in some conditions. The reference value for MPV is accepted as 6-10 fl [12]. It is an indicator for platelet reactivity and function $[5,6]$. Large platelets are more active (enzymatic and metabolic) than small platelets and produce more thromboxane A2 [13]. Also, MPV is a physiological variable of haemostasis. Larger platelets are denser and contain more a-granules, which can release prothrombotic substances, including platelet factor 4, platelet-derived growth factor, and P-selectin, a chemotactic and mitogenic factor contributing to vascular neointimal proliferation [6-8]. Although the most precise methodology to measure MPV is still uncertain, it is a routinely available parameter in the outpatient or inpatient setting at a rel-

Table I. The causes of abdominal pain $(N=579)$

\begin{tabular}{|c|c|c|}
\hline Diagnosis & Number & Percentage \\
\hline Non-specific abdominal pain & 330 & 56 \\
\hline Peptic ulcers & 74 & 12.6 \\
\hline Cystitis & 74 & 12.6 \\
\hline Gastroenteritis & 27 & 4.6 \\
\hline Nephrolithiasis & 25 & 4.2 \\
\hline Inflammatory bowel disease & 12 & 2 \\
\hline Due to cancers of colon, pancreas, and gall bladder & 4 from each group & 0.7 \\
\hline $\begin{array}{l}\text { Dysmenorrhoea, gastrointestinal bleeding, cholecystitis, ovarian cancer, acute } \\
\text { pancreatitis }\end{array}$ & 3 from each group & 0.5 \\
\hline Stomach cancer, umbilical hernia & 2 from each group & 0.3 \\
\hline $\begin{array}{l}\text { Intra-abdominal intrauterine device familial Mediterranean fever ovarian cyst, pelvic } \\
\text { inflammatory disease cirrhosis, vaginal fistula }\end{array}$ & 1 from each group & 0.2 \\
\hline
\end{tabular}


Table II. Demographic and laboratory data from the two groups

\begin{tabular}{lccc} 
Diagnosis & $\begin{array}{c}\text { NSAP* } \\
(n=330)\end{array}$ & $\begin{array}{c}\text { Others } \\
(n=249)\end{array}$ & $\begin{array}{c}\text { Value } \\
\text { of } p\end{array}$ \\
\hline Age [years] & $49 \pm 20$ & $49 \pm 19$ & 0.832 \\
\hline Gender (F/M) & $186 / 144$ & $144 / 105$ & \\
\hline Leukocyte $\left[/ \mathrm{mm}^{3}\right]$ & $9673 \pm 6444$ & $9243 \pm 4847$ & 0.372 \\
\hline Haemoglobin $[\mathrm{g} / \mathrm{dl}]$ & $12.5 \pm 1.9$ & $12.7 \pm 1.9$ & 0.382 \\
\hline Platelets $\left[/ \mathrm{mm}^{3}\right]$ & 287866 & 291891 & 0.682 \\
& \pm 119661 & \pm 116291 & \\
\hline MPV [fl] & $8.03 \pm 1.4$ & $8.27 \pm 1.1$ & 0.025 \\
*NSAP - & &
\end{tabular}

atively low cost [7]. A positive or negative relationship between MPV and platelet count has been reported in many studies $[8,10]$. Increased MPV was found in older smoking patients, but not in non-smokers $[14,15]$. We have no information about MPV or platelet counts in our smoker patients.

The MPVs were $8.9 \pm 1.4 \mathrm{fl}$ in the healthy population [16]. In our study, MPV values were $8.03 \pm 1.4 \mathrm{fl}$ in the NSAP group and $8.27 \pm 1.1 \mathrm{fl}$ in the other group. The most important problem regarding the clinical validity of MPV is that it changes over time as platelets swell in EDTA, with an increase of $7.9 \%$ within 30 min and an overall increase of $13.4 \%$ over $24 \mathrm{~h}$. However, the majority of this increase occurs in the first $6 \mathrm{~h}$ [17]. It is widely accepted that platelet swelling in test tubes can be minimised by rapid processing of samples, i.e. within $1 \mathrm{~h}$ [18]. Our study was performed in an emergency service, so whole blood counts were performed within $30 \mathrm{~min}$.

There are many retrospective studies about MPV values in the literature. Özlü et al. reported elevated MPV levels in patients with non-ST elevation acute coronary syndrome (NSTE-ACS) compared with control subjects in the young population [19]. Also, increased MPV was established to be an independent predictor of NSTE-ACS in their study [19]. In another study, it was shown that patients with coronary artery ectasia and coronary artery disease have higher MPVs than subjects with normal coronary angiograms [20].

Increased MPVs were reported in patients with obstructive sleep apnoea syndrome (OSAS), and this was related to severity of disease. Also, there is increased inflammation in OSAS [21]. Karaman et al. [22] reported lower MPVs in patients with non-functional pancreatic neuroendocrine tumour compared with pancreatic adenocarcinoma. However, many previous studies reported decreased MPV with inflammation. Decreased MPV in patients with active rheumatic arthritis and ankylosing spondylitis was reported [23]. Beyazit et al. [24] reported decreased MPV in patients with acute pancreatitis and increased MPV after treatment. The MPV was $7.8 \mathrm{fl}$ in our three patients with acute pancreatitis.

Increased MPV was reported in patients with IBS, compared to controls. This increase in MPV was independent of the type of irritable bowel syndrome [25]. The MPV was lower in patients with active inflammatory bowel disorders in the study by Jaremo and Sandberg-Gertzen [26]. The mean MPV value in 12 patients was $8.12 \mathrm{fl}$. Increased MPV is associated with cardio-cerebrovascular diseases or low-grade inflammatory conditions such as arterial or venous thrombosis. Familial Mediterranean fever and active rheumatoid arthritis were also found to be associated with lower levels of MPV [11].

Two principal technologies are used to size platelets: aperture impedance and flow cytometry laser optics. The outcome of these tests may have different results. We could not analyse MPV or platelet counts in smoking patients. Also, the current study was retrospective, without any control group. Several variables such as temperature, osmolarity, and $\mathrm{pH}$ may affect MPV. Prospective studies are required in larger groups to confirm our data.

\section{Conclusions}

Increased or decreased MPV may be associated, without any evidence change, with platelet or WBC counts. The MPV may be a useful, simple, and relatively cheap test to diagnose or differentiate between NSAP and others.

\section{Conflict of interest}

The authors declare no conflict of interest.

\section{References}

1. Fields JM, Dean AJ. Systemic causes of abdominal pain. Emerg Med Clin North Am 2011; 29: 195-210.

2. Irvin TT. Abdominal pain: a surgical audit of 1190 emergency admissions. Br J Surg 1989; 76: 1121-5.

3. McNamara R, Dean AJ. Approach to acute abdominal pain. Emerg Med Clin North Am 2011; 29: 159-73.

4. Mladeımov E, Granegger S, Hann S, et al. Platelet labeling for determination of lifespan. Türk J Haematol 2002; 19: 275-81.

5. Aksu K, Donmez A, Keser G. Inflammation-induced thrombosis: mechanisms, disease associations and management. Curr Pharm Des 2012; 18: 1478-93.

6. Bath PM, Missouris CG, Buckenham T, et al. Increased platelet volume and platelet mass in patients with atherosclerotic renal artery stenosis. Clin Sci 1994; 87: 253-7.

7. Leader A, Pereg D, Lishner M. Are platelet volume indices of clinical use? A multidisciplinary review. Ann Med 2012; 44: 805-16.

8. Chu SG, Becker RC, Berger PB, et al. Mean platelet volume as a predictor of cardiovascular risk: a systematic review and meta-analysis. J Thromb Haemost 2010; 8: 148-56. 
9. Uysal P, Tuncel T, Olmez D, et al. The role of mean platelet volume predicting acute exacerbations of cystic fibrosis in children. Ann Thorac Med 2011; 6: 227-30.

10. Yazici S, Yazici M, Erer B, et al. The platelet indices in patients with rheumatoid arthritis: mean platelet volume reflects disease activity. Platelets 2010; 21: 122-5.

11. Gasparyan AY, Ayvazyan L, Mikhailidis DP, et al. Mean platelet volume: a link between thrombosis and inflammation? Curr Pharm Des 2011; 17: 47-58.

12. Van der Loo B, Martin JF. Megakaryocytes and platelets in vascular disease. Baillieres Clin Haematol 1997; 10: 109-23.

13. Thompson CB, Eaton KA, Princiotta SM, et al. Size dependent platelet subpopulations: relationship of platelet volume to ultrastructure, enzymatic activity, and function. $\mathrm{Br}$ J Haematol 1982; 50: 509-19.

14. Kario K, Matsuo T, Nakao K. Cigarette smoking increases the mean trombosit volume in elderly patients with risk factors for atherosclerosis. Clin Lab Haematol 1992; 14: 281-7.

15. Arslan E, Yakar T, Yavasoglu I. The effect of smoking on mean platelet volume and lipid profile in young male subjects Anadolu Kardiyol Derg 2008; 8: 422-5.

16. Demirin H, Ozhan H, Ucgun T, et al. Normal range of mean platelet volume in healthy subjects: insight from a large epidemiologic study. Thromb Res 2011; 128: 358-60.

17. Bowles KM, Cooke LJ, Richards EM, et al. Platelet size has diagnostic predictive value in patients with thrombocytopenia. Clin Lab Haematol 2005; 27: 370-3.

18. Dastjerdi MS, Emami T, Najafian A, Amini M. Mean platelet volume measurement, EDTA or citrate? Hematology 2006; 11: 317-9.

19. Özlü MF, Öztürk S, Ayhan SS, et al. Predictive value of mean platelet volume in young patients with non-STsegment elevation acute coronary syndromes: a retrospective observational study. Anadolu Kardiyol Derg 2013; 13: 57-61.

20. Demir S, Avsar Mk, Karakaya Z, et al. Increased mean platelet volume is associated with coronary artery ectasia. Postep Kardiol Inter 2013; 9: 241-5.

21. Nena E, Papanas N, Steiropoulos P, et al. Mean platelet volume and platelet distribution width in non-diabetic subjects with obstructive sleep apnoea syndrome: new indices of severity? Platelets 2012; 23: 447-54.

22. Karaman K, Bostanci EB, Aksoy E, et al. The predictive value of meanplateletvolume in differential diagnosis of non-functional pancreatic neuroendocrine tumors from pancreatic adenocarcinomas. Eur J Intern Med 2011; 22: 95-8.

23. Kisacik B, Tufan A, Kalyoncu U, et al. Mean platelet volume (MPV) as an inflammatory marker in ankylosing spondylitis and rheumatoid arthritis. Joint Bone Spine 2008; 75: 291-4.

24. Beyazit Y, Sayilir A, Torun S, et al. Mean platelet volume as an indicator of disease severity in patients with acute pancreatitis. Clin Res Hepatol Gastroenterol 2012; 36: 162-8.

25. Aktas G, Alcelik A, Tekce BK, et al. Red cell distribution width and mean platelet volume in patients with irritable bowel syndrome. Prz Gastroenterol 2014; 9: 160-3.

26. Jaremo P, Sandberg-Gertzen $H$. Platelet density and size in inflammatory bowel disease. Thromb Haemost 1996; 75: 560-1.

Received: 8.08.2014

Accepted: 18.11.2014 